\title{
Double stenting technique for under-expanded stent due to rigid lesion
}

\author{
Hamid A. Abdulsada ${ }^{1}$
}

\begin{abstract}
Coronary stent under expansion is a strong risk factor for stent thrombosis and cardiac events. And once occurred it is a challenging condition, due to the fact that no uniform acceptance about how is the best strategy to solve the problem, the literature review summarize some cases that respond to cutting balloon, rotational atherectomy, laser therapy after failure of high pressure ballooning by noncompliant balloon, I report a case of stent under expansion treated successfully using a high pressure inflated second stent after failing repeated attempt to dilate the lesion by single and double balloons

The learning object: stent under expansion frequently observed in the setting of calcified rigid lesion, however this can be prevented to great extent by adequate lesion preparation. Once occurred it should be managed aggressively according to the facility of the cath lab, and experience of operator.
\end{abstract}

\section{INTRODUCTION}

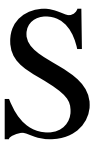

tent under-expansion may be multifactorial, but the presence of heavy calcium burden in plaques remains a significant cause, however, the calcium burden in certain plaques may be underappreciated during coronary angiography, complicating the procedure. ${ }^{[1]}$ It may be a challenge and dilemma exists on how best to deal with this clinical situation, Ideally the operators should take every necessary precaution to prevent the situation of an under deployed stent because Under-expansion of coronary stent represents an increased risk of instent restenosis and also predispose patients to stent thrombosis. These can lead to adverse cardiac events including acute coronary syndromes, myocardial infarction, and death. ${ }^{[2]}$ Fetuin-A (eg, 2-HeremensSchmid glycoprotein) is an abundant serum glycoprotein that binds calcium and phosphate in the circulation, thereby forming "calciprotein particles" that help clear the circulation of excess $\mathrm{Ca} \times \mathrm{PO}_{4}$, Low fetuin-A levels correlate with a chronic inflammatory state and cardiovascular calcification in hemodialysis patients, Computed tomography (CT) scan also detects and quantifies vascular calcification but does not differentiate between intimal and medial calcium deposition. ${ }^{[3]}$

\section{Case report}

A sixty - year old lady hypertensive not diabetic, with history of renal impairment, present with unstable angina, Canadian Cardiovascular Society (CCS) Class III symptoms despite full antianginal treatment. Her electrocardiography result shows left bundle branch block, echocardiography study shows mild concentric left ventricular hypertrophy and inferolateral wall hypokinesia, the left systolic function was normal, her blood urea of $80 \mathrm{mg} / \mathrm{dl}$, serum creatinine of $1.75 \mathrm{mg} / \mathrm{dl}$, she had resistant hypertension, coronary angiography shows intermediate lesion involving proximal left anterior descending artery (LAD), no critical circumflex (CX), dominant big right coronary artery (RCA) with critical mid lesion, distal intermediate lesion, although no apparent calcification seen by angiographic study and it was a vital artery and we elected to perform percutaneous coronary intervention (PCI) to the RCA.

\section{Procedure}

After adequate rehydration for three hours with normal saline, the RCA was wired with balanced middle weight wire, and after predilatation with balloon $3.0 * 20$ up to 14 barrs, which shows acceptable expansion (Figure $1 \mathrm{~A}, \mathrm{~B}$ ) then a stent promuse element $3.5 * 24$ was deployed with inflation pressure up to 15 barrs resulting in inadequate expansion at the mid Zone of the stent, additional Inflation using other balloon NC quntum $3.5 * 18$ up to 18 barrs failed to dilate the stent further, the patient experience severe chest pain during inflation for 20 second, with ST elevation at lead 3, and avf, though respond well to deflation of the balloon and use of intracoronary angesid, multiple inflation was done after the use of intracoronary angesid. Three attempt all failed to dilate the lesion, additional steps using simultaneous two short balloon inflations $\left(3.0^{*} 12 \mathrm{~mm}\right.$ in diameter) were inflated to 12,14 
(Figure 2.A, B) also failed to adequately expand the stent and resulting in return of chest pain and reelevation in inferior ECG leads. Lastly I thought of trial to deploy additional short stent over the under expanded segment to focus the pressure in that area., a stent $3.5^{*} 14$, initial inflation $p$ to 16 barrs fail but with inflation to very high pressure 20 barrs for short period resulting in full expansion of the lesion and relieve of stenotic area, the patient shows a relieve of her symptoms of chest pain, with stable vital sign (Figure $3 \mathrm{~A}, \mathrm{~B}$ ). The patient discharged next day in stable condition after adequate rehydration for 12 hours after the procedure. During follow up mild elevation of her serum creatinine that decrease gradually and return to baseline after two weeks. Reassessment after three months follow up, her symptom decrease dramatically and is currently CCS functional class 1 .

\section{DISCUSSION}

Stent under expansion should always be avoided, and still a challenging hurdle to overcome despite current advances in the modern stent era. There is limited evidence for best practice in this situation and others have previously reported similar situations as ours similar clinical outcomes, Ideally the operators should take every necessary precaution to prevent the situation of an under deployed stent, including ensuring adequate predilatation and ensuring sufficient balloon expansion, and also using IVUS to better understand the lesion anatomy as calcium burden may easily be underestimated. Rotablation can then be performed as necessary, prior to stent implantation. ${ }^{[4]}$ As suggested by Sunew et al in cases of undilatable, under expanded stents due to rigid, heavily calcified lesions, the options are limited when high-pressure balloon inflations with shorter, noncompliant balloons fail to successfully expand the stents. The options include modalities such as rotational atherectomy, excimer laser, and cutting balloons. ${ }^{[5]}$ Rotational atherectomy of lesions is an alternative method to facilitate PCI, when routine balloon angioplasty fails to successfully dilate the lesion. High-speed rotational atherectomy preferentially cuts hard plaque, increasing plaque compliance, thereby rendering the lesion more amenable to balloon dilatation. Therefore, to facilitate stent expansion inheavily calcified lesions, rotational atherectomy prior to stent implantation is often required. Kobayashi, et al reported a case in which a stent was implanted in a heavily calcified "napkin-ring" lesion resulting in marked stent under expansion. When in-stent restenosis developed, intravascular ultrasound revealed the predominant cause of the stenosis was the under expanded stent within a calcified band. When high-pressure balloon angioplasty failed to dilate the lesion, ablation of the stent struts using stentablation was performed (Rotational atherectomy). ${ }^{[6]}$ Another report of similar management by vales et al who report a case via the transradial approach of rotationalatherectomy facilitated PCI of in-stent restenosis of a everely under expanded stent due to a heavily calcifiedPlaque, ${ }^{[7]}$ however although limited data about the complication of rotational atherectomy, Little information is available regarding the size of metallic embolic particles and the likelihood of adverse clinical consequences. Another possible complication from stent ablation is sub acute or late stent thrombosis because some stent struts may become irregular or damaged and contact blood flow directly after rotational atherectomy. ${ }^{[8]}$ The Cutting Balloon (CBA) has also been used to treat resistant lesions. This catheter has 3-4 atherotomes (microsurgical blades) longitudinally attached to the balloon, with which balloon inflation induces cutting or incision of the atherosclerotic plaque. These microtomes, approximately $0.25 \mathrm{~mm}$ in height, are 3-5 times sharper than conventional surgical blades. The microtome edge initiates an indentation into the plaque, after which the shear force applied by the balloon inflation propagates the crack, Kang WC et al reported a case in which direct stenting was done in mid Right Coronary Artery (RCA) resulting in under expansion of the stent due to calcification in the lesion that was not apparent initially. They used incremental upsizing of CBA and started with small diameter cutting balloon initially and then replaced by larger ones and got the successful expansion of stent. ${ }^{[9]}$ Muhammad, et al report a case in which a calcification in mid left anterior descending (LAD) artery was not apparent initially on angiogram and stenting was done after inappropriate predilation resulting in under expansion of stent. High pressure inflation, buddy wire technique, scoring and cutting balloon inflation failed to achieve the full expansion of stent and patient was sent for minimally invasive direct coronary artery bypass graft (MIDCAB) surgery. ${ }^{[10]}$ Various complications related to CBA have been reported including arterial rupture, 
delayed perforation, stent strut avulsion, and fracture of microsurgical blades, however, very few cases have been reported. Regarding inadvertent stent extraction as a complication of CBA. ${ }^{[1]}$ Eximer laser ablation was considered as another alternative to treat the lesion. However, laser angioplasty is not as efficient at ablating calcified plaque (and pruning stent struts) as rotational atherectomy. ${ }^{[4]}$ All the mentioned procedure require additional stent after doing cutting or ablation of the under expanded stent with their risk of ablation of the elution substance. I had use many trial of balloon inflation for under expanded stent and inflation mostly shows changes at the periphery of the balloon leaving the center of the balloon, this procedure also fail after even using two balloon simultaneously, then when I use a second stent in the narrowed area of stent under expanded with inflation pressure very high for short second makes the focus of pressure more over that under expanded area and the result was fully expanded stent.

\section{CONCLUSION}

It is not uncommon to had a under expanded stent in coronary intervention, although most respond to overinflating, but is rather rare to had resistant under expanded stent and in all method of management of this condition whether rotational ablation, cutting balloon all use second stent after expansion of previous stent. So our technique of a second short stent inside the unexpanded segment will not give additional stent more than other methods used in the literature, although it was successful in overcoming our problem probably by focusing inflation pressure over the unexpanded part and prevent recoil of the artery.

\section{I have no conflict of interest}

\section{REFERENCE}

1. Uren NG, Schwarzacher SP, Metz JA, Lee DP, Honda $Y$, et al. Predictors and outcomes of stent thrombosis: an intravascular ultrasound registry. Eur Heart J, 2002; 23: 124-132

2. Cheneau E, Leborgne L, Mintz GS, Kotani J, Pichard AD, et al. Predictors of sub acute stent thrombosis: results of a systematic intravascular ultrasound study. Circulation, 2003; 108: 43-47.

3. Cozzolino M, Galassi A, Biondi ML, et al. Serum fetuin-A levels link inflammation and cardiovascular calcification in hemodialysis patients. Am J Nephrol 2006; 26: 423.

4. Lee S, Park KW, Kim HS. Stent ablation of an under expanded stent in a heavily calcified lesion using rotational atherectomy. J Cardiovasc Med (Hagerstown) 2010; 13: 284-288.

5. Sunew J, Chandwaney RH, Stein DW, Meyers S, Davidson CJ. Excimer laser facilitated percutaneous coronary intervention of a nondilatable coronary stent. Catheter Cardiovasc Interv 2001; 53(4): 513-517, discussion 518

6. Kobayashi Y, Teirstein PS, Linnemeier TJ, Stone GW, Leon MB, Moses JW. Rotational atherectomy (stentablation) in a lesion with stent under expansion due to heavily calcified plaque. Catheter Cardiovasc Interv 2001; 52(2): 208-211

7. Vales L, Coppola J, Kwan T. Successful expansion of an under expanded stent by rotational atherectomyInt J Angiol. 2013; 22(1):63-68.

8. Mehran R, Dangas G, Mintz GS, Waksman R, Abizaid A, SatlerLF, Pichard AD, Kent KM, Lansky AJ, Stone GW, Leon MB. Treatment of in-stent restenosis with excimer laser coronary angioplasty versus rotational atherectomy: comparative mechanisms and results. Circulation 2000; 101: 2484 -2489.

9. Kang WC, Ahn TH, Han SH, Shin EK. Successful management of a resistant, focal calcified lesion following direct coronary stenting with a cutting balloon. J Invas Cardiol 2004; 16: 725-726.

10. Muhammad T Farman, Jawaid A Sial, Naveed U Khan. Undefeatable coronary lesion. J Pak Med Assoc. 2011; 61(2): 185-187.

11.Haridas KK, Vijayakumar M, Viveka K, Rajesh $T$, Mahesh NK. "Fracture of cutting balloon microsurgical blade inside coronary artery during angioplasty of tough restenotic lesion: a case report," Catheterization and Cardiovascular Interventions, vol. 58, no. 2, pp. 199-201, 2003. 


\section{Figure legends}

Fig 1. The left angiographic picture shows the mid RCA lesion critical at the mid although no apparent calcification seen by angiographic study, the right shows the lesion after dilatation using balloon (3.0*20) up to 14 bars, although acceptable but not fully dilated

Fig 2. The left shows angiographic picture after high pressure inflation in the stent $(3.5 * 24)$ reaching 15 bars, and additional ballooning 18 bars, the lesion dilated at the periphery although still not dilated at the mid, the right shows two balloon inflation $(3.0 * 12)$ to high pressure also fail to dilate the lesion

Fig 3. Shows the angiographic result after high pressure inflation inside the short stent $(3.0 * 14)$ successfully dilate the under expanded stent, the right side shows the final angiographic picture with fully dilated stent

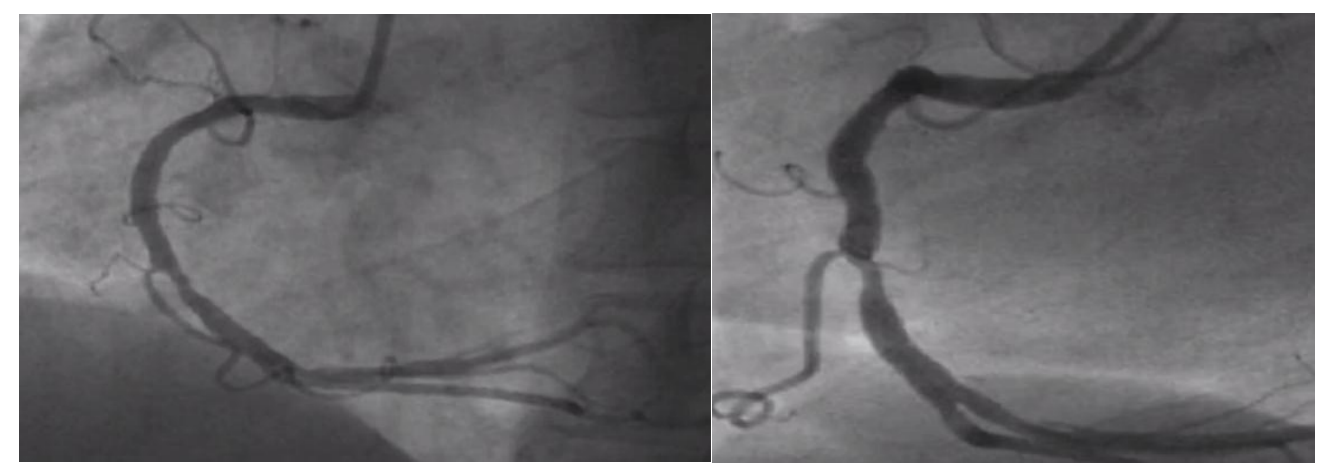

Fig 1. The left angiography shows the mid RCA lesion, the right shows result after ballooning

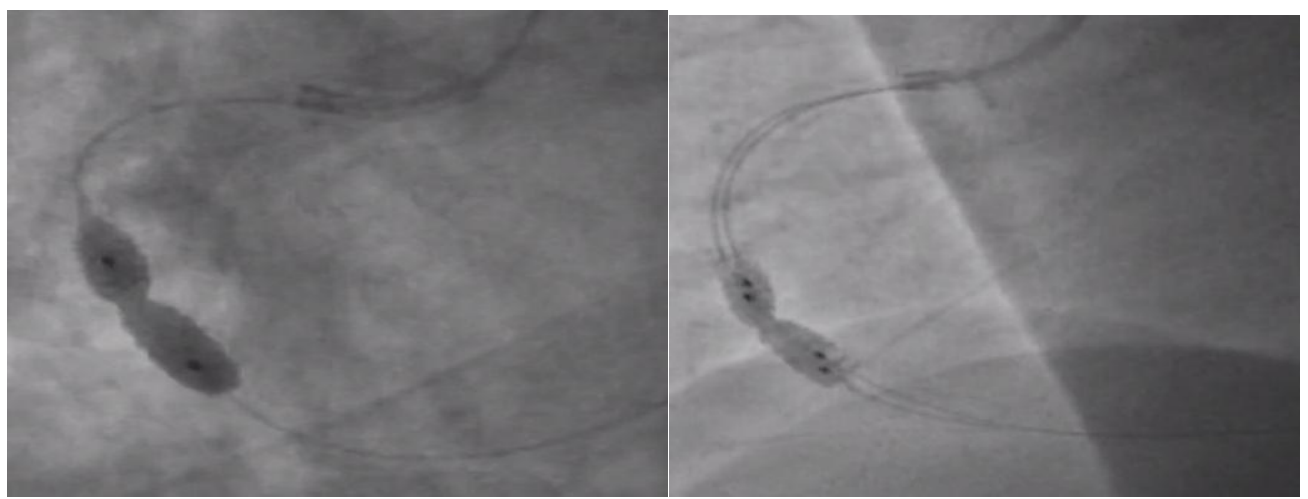

Fig 2. The left angio shows high pressure single ballooning, the right shows two ballooning

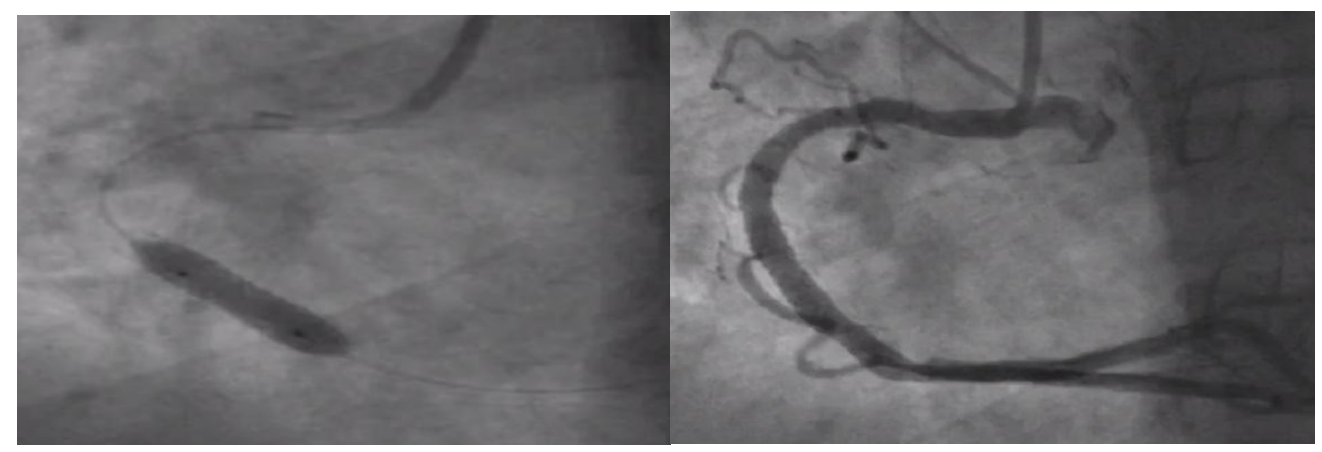

Fig 3. The left angio shows high inflation in second stent inside the first one, the right shows final result 